Gastrointestinal complications

\section{Complications of gastrointestinal endoscopy}

\section{Kevin R Palmer}

\section{Scoping our practice}

$\mathrm{T}$ he benefits of endoscopy have increased enormously as it has matured from a purely diagnostic tool to become a therapeutic subspecialty, but so too has the potential for causing harm. Endoscopic haemostasis for varices and ulcers, relief of biliary obstruction from stones or tumours, and alleviation of luminal obstruction using balloons, bougies and stents improve quality of life and may obviate the need for operative surgery, but all risk serious complications. The risks of endoscopy are worth taking only when the procedure is worth doing and when the benefits outweigh the risks. Complications are unacceptable if endoscopy is not indicated; pancreatitis after diagnostic endoscopic retrograde cholangiopancreatography (ERCP) and colonoscopic perforation done during follow-up of a single rectosigmoid polyp are examples. The risk:benefit ratio is dictated by the disease process and by the underlying health of the patient-thus (to rehearse an old chestnut), the complications of endoscopic bile duct clearance are probably fewer and better tolerated than those of surgical exploration in the elderly and frail and are therefore acceptable; the same may not be true for younger and fitter patients for whom surgery has a lower complication rate than ERCP. Relatively high-risk endoscopic procedures can be justified to palliate symptoms in patients with terminal diseases, whereas the risks become unacceptable when the same procedure is done to a patient with surgically respectable disease.

These issues received considerable airing in the National Confidential Enquiry into Patient Outcome and Death report "scoping our practice", ${ }^{1}$ which analysed death occurring within 30 days of therapeutic endoscopy in the UK. National Confidential Enquiry into Patient Outcome and Death had previously dealt with complications of operative surgery and has undoubtedly altered the processes with benefit for patients undergoing emergency surgery. "Scoping our practice" reviewed 1818 therapeutic procedures undertaken in 263 hospitals with an analysis of events that contributed to complications and death. The project can be criticised; a large number of therapeutic procedures that were undertaken in outpatients over the same time period were not considered, reporting was voluntary, analysis was subjective and the project could in no way be regarded as a formal audit. Obviously, most of the deaths were not a consequence of complications and almost all patients were elderly, many had advanced malignant disease and most had serious medical comorbidities. Nevertheless, the large number of case histories that form the basis of the report is compelling; particularly, as it is likely that for every complication that led to death, several "near misses" were probable. "Scoping our practice" highlighted a range of inadequacies, which were associated with the occurrence of complications. These included organisational aspects, particularly lack of emergency cover for patients admitted with acute gastrointestinal bleeding in many hospitals, the appropriateness of procedures (the "futility" of undertaking percutaneous endoscopic gastrostomy in terminally ill patients) and specific aspects of endoscopy including unsafe sedation, lack of anaesthetic support in unstable patients and some technical practices. The report recommended that there should be national guidelines to ensure competency in endoscopy. In fact, many guidelines do exist The British Society of Gastroenterology has produced guidelines for the management of acute gastrointestinal bleeding, ${ }^{2}$ acute pancreatitis, ${ }^{3}$ bleeding from portal hypertension, ${ }^{4}$ cleaning and disinfection of endoscopes, ${ }^{5}$ oesophageal dilatation, among others. In addition, the Joint Advisory Group for Gastrointestinal Endoscopy (JAG) guidelines ${ }^{7}$ define criteria for safe endoscopy (including sedation guidelines, consent, observations of patients during and after endoscopy, technical aspects for a whole range of diagnostic and therapeutic procedures) and have sections relating to training.

Accepting that complications will inevitably occur, as illustrated by "scoping our practice", it is beholden to only undertake procedures that are clinically indicated and to act in a way that maximises the chances of success and minimises the risks of complications. Intuitively, a procedure is safer if done by an expert rather than by a novice. It also goes without saying that all involved in therapeutic endoscopy must be properly trained. There have, in recent years, been undoubted improvements in the quality of endoscopy and in training of the workforce in the UK. The colonoscopy audit undertaken in England in the $1990 \mathrm{~s}^{8}$ showed disturbing levels of performance including an average coaecal intubation rate of only $57 \%$ and a colon perforation rate of 1:757. Since then, there has been a sea change in training, stimulated by the modernisation agency endoscopy programme and subsequently by the requirements to deliver high quality colonoscopy as part of the colon cancer screening programme. Training courses in basic endoscopy, colonoscopy and "train the trainer" have been delivered widely throughout England and have helped develop a culture of training and learning. The "Global Rating Scale" introduced by Roland Valori et al has focused attention on all aspects of the patient journey throughout the endoscopy process, and serial Global Rating Scale scores have shown improvements in outcome in most of the units. It is clear therefore that the workforce is becoming better trained and that this is translating into improved performance including reduced risk for patients.

One of the responses of the British Society of Gastroenterology to "scoping our practice" has been to commission a working party to deal with complications of endoscopy, and this is published online on the BSG website. ${ }^{9}$ The report focuses on the important high-risk areas of sedation, therapeutic upper endoscopy, ERCP, percutaneous endoscopic gastrostomy insertion and colonoscopy. The frequency and causes of complications are described; approaches to diagnosis and treatment are defined. Clearly, there are difficulties in developing the evidence base for many of the conclusions, as randomised control trials are relatively few and the recommendations are therefore generally grade C. Nevertheless, the reviews are exhaustive, recommendations (in places pragmatic, but common sense) are robust and authors must be congratulated. All involved in endoscopy should read and digest the report.

What should happen to "complications of endoscopy"? One would hope that it will not lie dusty on the shelf in the unit coffee room and that it will be regularly electronically updated. Its value may lie 
partly in helping individual units construct patient information and consent forms. It will be useful in creating audit standards for units, for individual trainees and established endoscopists. I would like the report to be used in a highly constructive manner to help improve the quality assurance of endoscopy. The JAG has quality assured $>250$ endoscopy units for training and this has been credible as JAG represents all the relevant stakeholders (the Royal Colleges, Specialist Societies, nurses, etc). It is now appropriate to widen the JAG remit beyond training standards to one of quality assurance for the endoscopy service. This has already effectively happened for units undertaking colon cancer screening colonoscopy in England where a successful JAG accreditation visit is a mandatory requirement. The JAG is in the ideal quality assurance body for all endoscopy units, irrespective of whether or not they contribute to the screening programme. Quality assurance needs auditable standards and "complications of endoscopy" is an excellent text from which to develop safety standards. Working party reports like this one, are powerful tools in our quest to maintain and improve quality, we should use them to their greatest potential.

Gut 2007;56:456-457.

doi: $10.1136 /$ gut.2006.105577

Correspondence to: Kevin R Palmer, Western General Hospital, Crewe Road, Edinburgh EH4 2XU, UK; kpalmer@golf5063.freeserve.co.uk

Competing interests: None.

\section{REFERENCES}

1 Scoping our Practice. The 2004 report of the National Confidential Enquiry into Patient Outcome and Death. London: NCEPOD, 2005.
2 Non-variceal upper gastrointestinal haemorrhage: guidelines. Gut 2002;51(Suppl 4):iv1-iv6.

3 UK Working Party on Acute Pancreatitis. UK guidelines for the management of acute pancreatitis. Gut 2005;54(Suppl 3):iiil-iii9.

4 Jalan R, Hayes PC. UK guidelines on the management of variceal haemorrhage in cirrhotic patients. British Society of Gastroenterology. Gut 2000;46(Suppl 3-4):III1-III15.

5 BSG Endoscopy Committee Working Party. Cleaning and disinfection of equipment for gastrointestinal endoscopy. Report of a working party of the British Society of Gastroenterology Endoscopy Committee Gut 1998:42.585-93.

6 Riley SA, Attwood SE. Guidelines on the use of oesophageal dilatation in clinical practice. Gut 2004;53(Suppl 1):i1-i6.

7 JAG. Guideline for the training, appraisal and assessment of trainees in gastrointestinal endoscopy and for the assessment of units for registration and re-registration 2004. http://www.thejag.org.uk/ see "Guidelines" (accessed 5 Jan 2006).

8 Bowles CJA, Leicester R, Romaya C, et al. A prospective study of colonoscopy practice in the UK today: are we adequately prepared for national colorectal cancer screening tomorrow? Gut 2004:53:277-83

9 Guidelines on Complications of Gastrointestinal Endoscopy. See "Guidelines Index" 2006 (accessed 5 Jan 2006). http://www.bsg.org.uk/.

\section{Helicobacter pylori and oesophageal cancer-not always protective}

\section{Kenneth E L McColl}

\section{The mechanism of the positive association between atrophic gastritis and oesophageal squamous cell carcinoma}

$\mathrm{H}$ elicobacter pylori infection is now widely accepted to be an important risk factor for a number of gastric and duodenal disorders. It is the major aetiological factor for gastric and duodenal ulcers unrelated to NSAID or aspirinusage. In addition, it plays an essential role in the aetiology of most cases of noncardia gastric cancer as well as low-grade gastric MALTomas. Such is the importance of $H$ pylori infection in disorders of the stomach and duodenum that pathology distal to the gastro-oesophageal junction is rarely encountered when endoscoping $H$ pylori naive subjects.

Over the past few years, interest in $H$ pylori has extended from its role in the aetiology of diseases of the stomach and duodenum to its possible role in the aetiology of diseases of the oesophagus. This has focused largely on the possibility that $H$ pylori infection may protect against the development of gastro-oesophageal reflux disease and its complications of Barrett's oesophagus and oesophageal adenocarcinoma.
Most epidemiological studies have demonstrated a negative association between $H$ pylori infection and gastrooesophageal reflux disease or its complications. The prevalence of $H$ pylori infection is lower in reflux patients than in controls. ${ }^{1-3}$ There is also some evidence that reflux disease in $H$. pylori-negative patients tends to be more severe than in H. pylori-positive patients. ${ }^{4}$ In addition, more virulent, cytotoxin-associated gene A (cag A)-positive strains of $H$ pylori are associated with less severe reflux disease. $^{56}$ Numerous studies have now reported a strong negative association between $H$ pylori infection and risk of adenocarcinoma of the oesophagus or gastro-oesophageal junction. ${ }^{7-11}$ The strength of the negative association between $H$ pylori infection and reflux disease or its complications appears to be related to the countries in which the association has been studied, being more marked in the East than the West. ${ }^{12}$

The negative association observed in epidemiological studies raises the possibility of a protective effect of the infection on reflux disease but does not establish it. Such association could be explained by confounding factors, which are influencing both the prevalence of infection and prevalence of oesophageal disease. Interventional studies examining the effect of treating the infection on oesophageal disease have examined the nature of the association. Such studies have produced conflicting results, showing that treating the infection may increase, decrease or have no effect on reflux disease. ${ }^{13}{ }^{14}$ Just as an East-West divide is apparent with respect to the epidemiological relationship between $H$ pylori and reflux disease, a similar EastWest divide is apparent with respect to the effect of treating the infection on reflux disease. In the East, treating $H$ pylori aggravates GERD, but not in the West. ${ }^{15-18}$

Is there a biological explanation for the complex association between $H$ pylori and reflux disease and the variations in the association between different regions of the world? A plausible explanation lies in the complex effects which $H$ pylori infection exerts on gastric acid secretion, which is the most damaging factor in the development of reflux disease and its complications. $H$ pylori infection may increase, decrease or have no overall effect on acid secretion, and its effect depends upon the pattern of gastritis induced by the infection. ${ }^{19}$ In some patients, the infection produces an antral-predominant, non-atrophic gastritis and this increases the level of gastric acid secretion. This pattern of gastritis is seen in subjects who develop duodenal 